\title{
The Explosive Universe with Gaia
}

\section{Lukasz Wyrzykowski ${ }^{1,2}$, Simon T. Hodgkin ${ }^{2}$, Nadejda Blagorodnova $^{2}$ and Vasily Belokurov ${ }^{2}$}

${ }^{1}$ Warsaw University Astronomical Observatory, Al. Ujazdowskie 4, 00-478 Warszawa, Poland email: wyrzykow@astrouw.edu.pl

${ }^{2}$ Institute of Astronomy, University of Cambridge, Madingley Road, CB3 0HA Cambridge, UK emails: (wyrzykow, sth, nblago, vasily) @ast.cam.ac.uk

Abstract. The Gaia mission will observe the entire sky for 5 years providing ultra-precise astrometric, photometric and spectroscopic measurements for a billion stars in the Galaxy. Hence, naturally, Gaia becomes an all-sky multi-epoch photometric survey, which will monitor and detect variability with millimag precision as well as new transient sources such as supernovae, novae, microlensing events, tidal disruption events, asteroids, among others.

Gaia data-flow allows for quick detections of anomalies within $24-48 \mathrm{~h}$ after the observation. Such near-real-time survey will be able to detect about 6000 supernovae brighter than 19 mag up to redshifts of $\mathrm{Z} \sim 0.15$. The on-board low-resolution $(\mathrm{R} \sim 100)$ spectrograph will allow for early and robust classification of transients and minimise the false-alert rate, even providing the estimates on redshift for supernovae. Gaia will also offer a unique possibility for detecting astrometric shifts in microlensing events, which, combined with Gaia's and ground-based photometry, will provide unique mass measurements of lenses, constrains on the dark matter content in the Milky Way and possible detections of free floating black holes.

Alerts from Gaia will be publicly available soon after the detection is verified and tested. First alerts are expected early in 2014 and those will be used for ground-based verification. All facilities are invited to join the verification and the follow-up effort. Alerts will be published on a web page, via Skyalert.org and via emailing list. Each alert will contain coordinates, Gaia light curve and low-resolution spectra, classification and cross-matching results.

More information on the Gaia Science Alerts can be found here:

http://www.ast.cam.ac.uk/ioa/wikis/gsawgwiki/

The full version of the poster is available here: http://www.ast.cam.ac.uk/ioa/wikis/gsawgwiki/images/1/13/GaiaAlertsPosterIAUS298.pdf

Keywords. surveys, supernovae: general, novae, variables:other, gravitational lensing, methods: statistical, catalogs
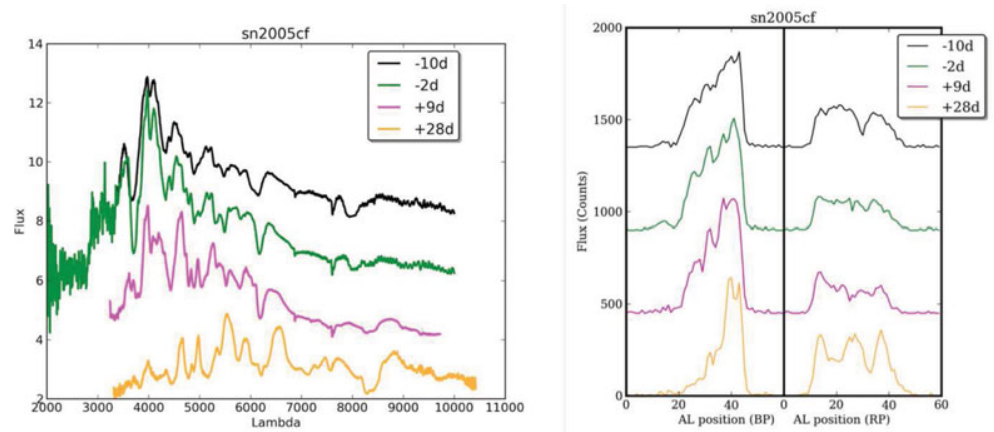

Figure 1. Simulation of Gaia low resolution spectra for different epochs of an example supernova type Ia. The spectra will allow for determining the type, redshift and epoch of most of the supernovae down to about 19 mag. Spectra simulated at GMag=17 using XpSim Gaia BpRp spectra simulator. A. Brown, private communicaiton. 